
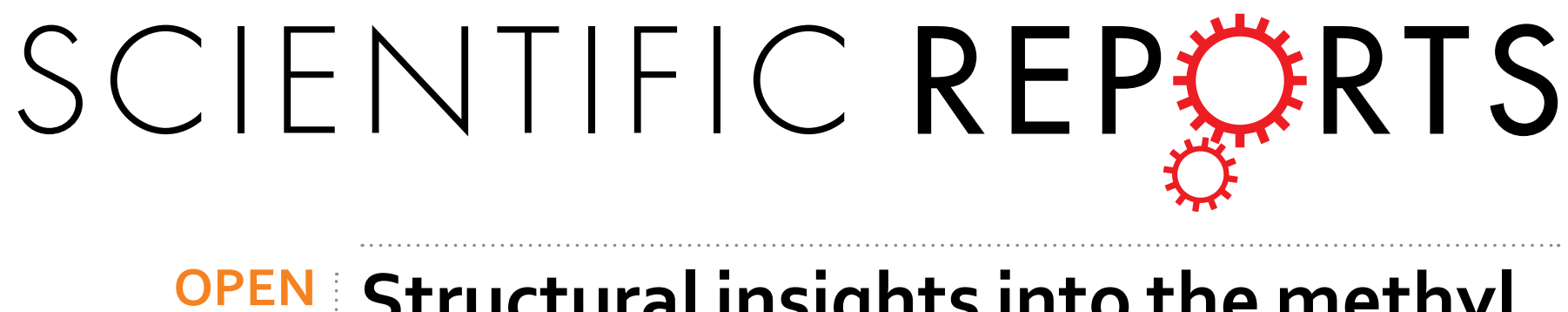

\title{
Structural insights into the methyl donor recognition model of a novel membrane-binding protein UbiG
}

Received: 25 October 2015

Accepted: 25 February 2016

Published: 15 March 2016
Yuwei Zhu ${ }^{1,2, *}$, Xuguang Jiang ${ }^{1,2, *}$, Chongyuan Wang ${ }^{1,2}$, Yang Liu ${ }^{1,2}$, Xiaojiao Fan ${ }^{1,2}$, Linjuan Zhang ${ }^{1,2}$, Liwen Niur, ${ }^{1,2}$ Maikun Teng ${ }^{1,2} \& \mathrm{Xu} \mathrm{Li}^{1,2}$

UbiG is a SAM-dependent $O$-methyltransferase, catalyzing two $O$-methyl transfer steps for ubiquinone biosynthesis in Escherichia coli. UbiG possesses a unique sequence insertion between $\beta 4$ and $\alpha 10$, which is used for membrane lipid interaction. Interestingly, this sequence insertion also covers the methyl donor binding pocket. Thus, the relationship between membrane binding and entrance of the methyl donor of UbiG during the $O$-methyl transfer process is a question that deserves further exploration. In this study, we reveal that the membrane-binding region of UbiG gates the entrance of methyl donor. When bound with liposome, UbiG displays an enhanced binding ability toward the methyl donor product S-adenosylhomocysteine. We further employ protein engineering strategies to design UbiG mutants by truncating the membrane interacting region or making it more flexible. The ITC results show that the binding affinity of these mutants to SAH increases significantly compared with that of the wildtype UbiG. Moreover, we determine the structure of UbiG $\Delta^{165-187}$ in complex with SAH. Collectively, our results provide a new angle to cognize the relationship between membrane binding and entrance of the methyl donor of UbiG, which is of benefit for better understanding the $O$-methyl transfer process for ubiquinone biosynthesis.

Ubiquinone (coenzyme Q), an essential lipid in the electron transport chain, is found in the inner mitochondrial membrane of eukaryotes as well as the plasma membrane of prokaryotes ${ }^{1,2}$. Ubiquinone plays a pivotal role in shuttling electrons from complex I or II to complex III for ATP synthesis in bacteria and higher eukaryotes ${ }^{3}$. In Homo sapiens, ubiquinone is tightly related to a number of diseases like muscular, cancer, diabetes and neurodegenerative disorders ${ }^{4-6}$. The biosynthesis of ubiquinone between prokaryotes and eukaryotes is similar, both beginning with the assembly of a quinone head group and a variable-length hydrophobic isoprenoid tail. Then, modifications of the benzoquinone are followed, including $C$-hydroxylation, decarboxylation, $O$-methylation and C-methylation ${ }^{7-9}$.

UbiG, a 240-residues protein in E. coli, is identified to be essential for ubiquinone biosynthesis in vivo. Mutations in the $u b i G$ gene could cause ubiquinone deficiency ${ }^{10}$. UbiG belongs to the Class I SAM-dependent-methyltransferases family, catalyzing the transfer of the methyl group from SAM to substrate ${ }^{11,12}$. In E. coli, the biosynthesis of ubiquinone needs two $O$-methylation steps, both of which are catalyzed by UbiG. The first $O$-methylation step is converting 2-polyprenyl-6-hydroxyphenol to 2-polyprenyl-6-methoxyphenol. The second step involves the $O$-methylation of 2-polyprenyl-3-methyl-5-hydroxy-6-methoxy-1,4-benzoquinol to form ubiquinone ${ }^{13}$.

Notably, unlike other types of methylation processes, the $O$-methylation reaction for ubiquinone biosynthesis in vivo is membrane associated ${ }^{14}$. The structure of full-length UbiG was determined and analyzed by our previous studies $^{15}$. UbiG exhibits a globular fold, and the core structure comprises eight-stranded $\beta$ sheet. Compared with the typical Class I SAM-dependent $O$-methyltransferases, UbiG possesses a unique sequence insertion shaping a membrane interaction patch. Meanwhile, our previous work indicated that UbiG binds preferentially to phosphatidylglycerol (PG) and cardiolipin (CL), two major components of E. coli plasma membrane, and the mutation compromising UbiG membrane interaction largely diminishes the growth rate of $E$. coli cells, revealing that the membrane-binding ability is pivotal for the function of UbiG in vivo ${ }^{15}$. Nevertheless, due to the lack of further

${ }^{1}$ Hefei National Laboratory for Physical Sciences at Microscale, Innovation Center for Cell Signaling Network, School of Life Science, University of Science and Technology of China, Hefei, Anhui, 230026, People's Republic of China. ${ }^{2}$ Key Laboratory of Structural Biology, Hefei Science Center of CAS, Chinese Academy of Science, Hefei, Anhui, 230026, People's Republic of China. *These authors contributed equally to this work. Correspondence and requests for materials should be addressed to M.T. (email: mkteng@ustc.edu.cn) orX.L. (email: sachem@ustc.edu.cn) 
<smiles>COc1cccc(C)c1O</smiles>

B
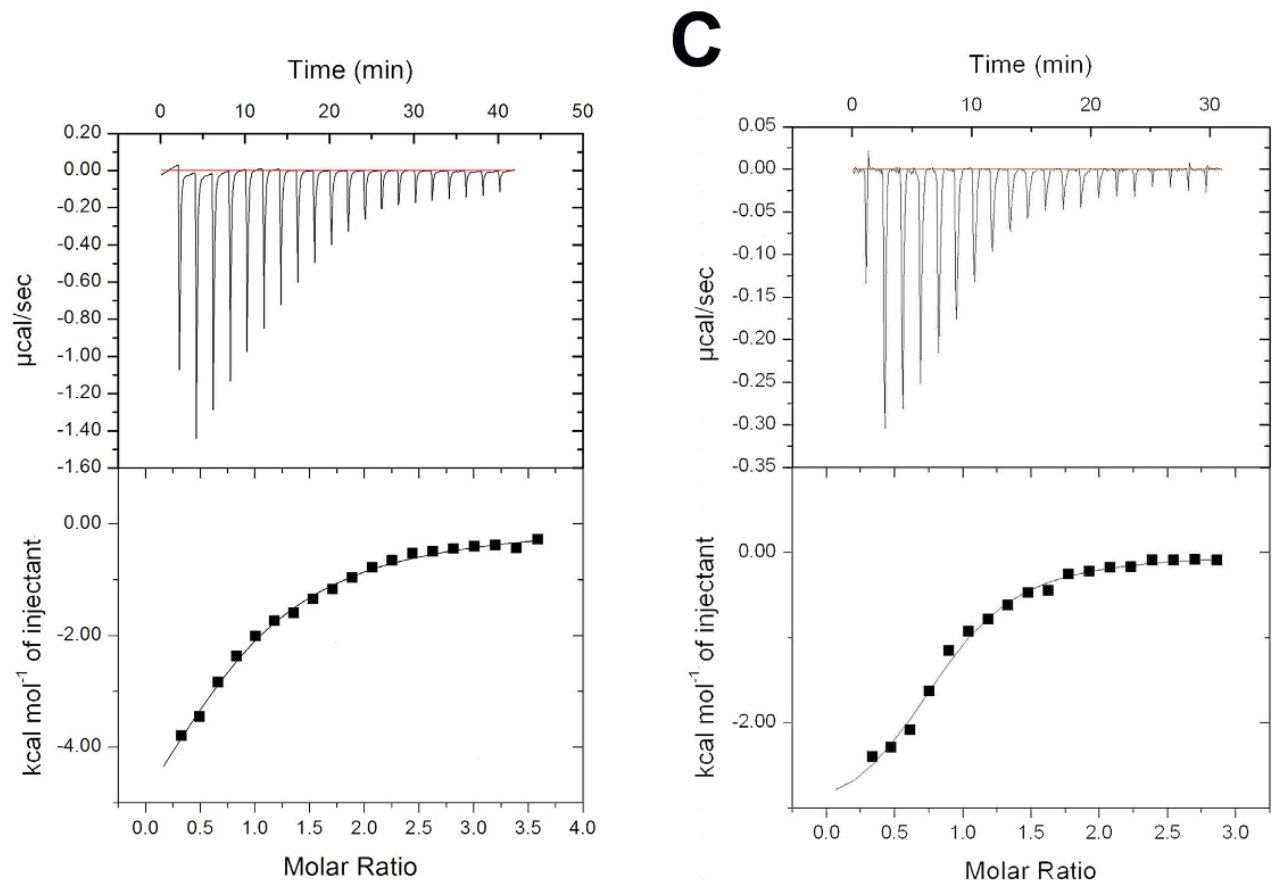

Figure 1. Membrane association promotes UbiG interacting with SAH. $O$-Methyltransferase reactions catalysed by UbiG in ubiquinone biosynthesis. (A) UbiG catalyzes two $O$-Methyltransferase steps in ubiquinone biosynthesis. The first $O$-methylation step is converting 2-polyprenyl-6-hydroxyphenol (compound 1) to 2-polyprenyl-6-methoxyphenol (compound 2). The second step involves the $O$-methylation of 2-polyprenyl-3methyl-5-hydroxy-6-methoxy-1,4-benzoquinol (compound 3) to form ubiquinone (compound 4). ITC profile of SAH titrated against wild-type $\mathrm{UbiG}(\mathbf{B})$ and liposome-bound $\mathrm{UbiG}(\mathbf{C})$. The upper panels showed the raw ITC data for injection of ligands into the sample cell containing wild-type UbiG or liposome-bound UbiG. The peaks were normalized to the ligand: protein molar ratio, and were integrated as shown in the bottom panels. Solid dots indicated the experimental data, and their best fit was obtained from a nonlinear least squares method, using a one-site binding model depicted by a continuous line.

structural information, the methyl donor recognition model of UbiG remains unclear. Furthermore, the significance of the membrane-binding ability of UbiG in the $O$-methyl transfer process for ubiquinone biosynthesis is still worth exploring.

Here, we construct an UbiG mutant (UbiG $\Delta^{165-187}$ ) by deleting the sequence insertion that covers the methyl donor binding pocket. The binding affinity of UbiG $\Delta^{165-187}$ to SAH is approximately 58 -fold higher than that of wild-type UbiG. Moreover, both wild-type UbiG bound to liposome and UbiG mutants that weaken the interaction of this sequence insertion with the core component show an enhanced binding ability toward SAH. Finally, we solve the crystal structure of UbiG $\Delta^{165-187}$ complexed with SAH at $2.10 \AA$. Taken together, our results uncover 


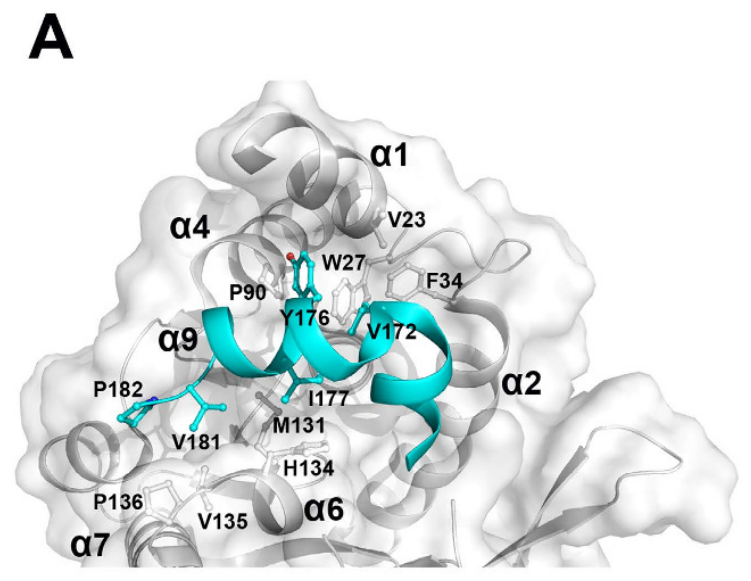

B
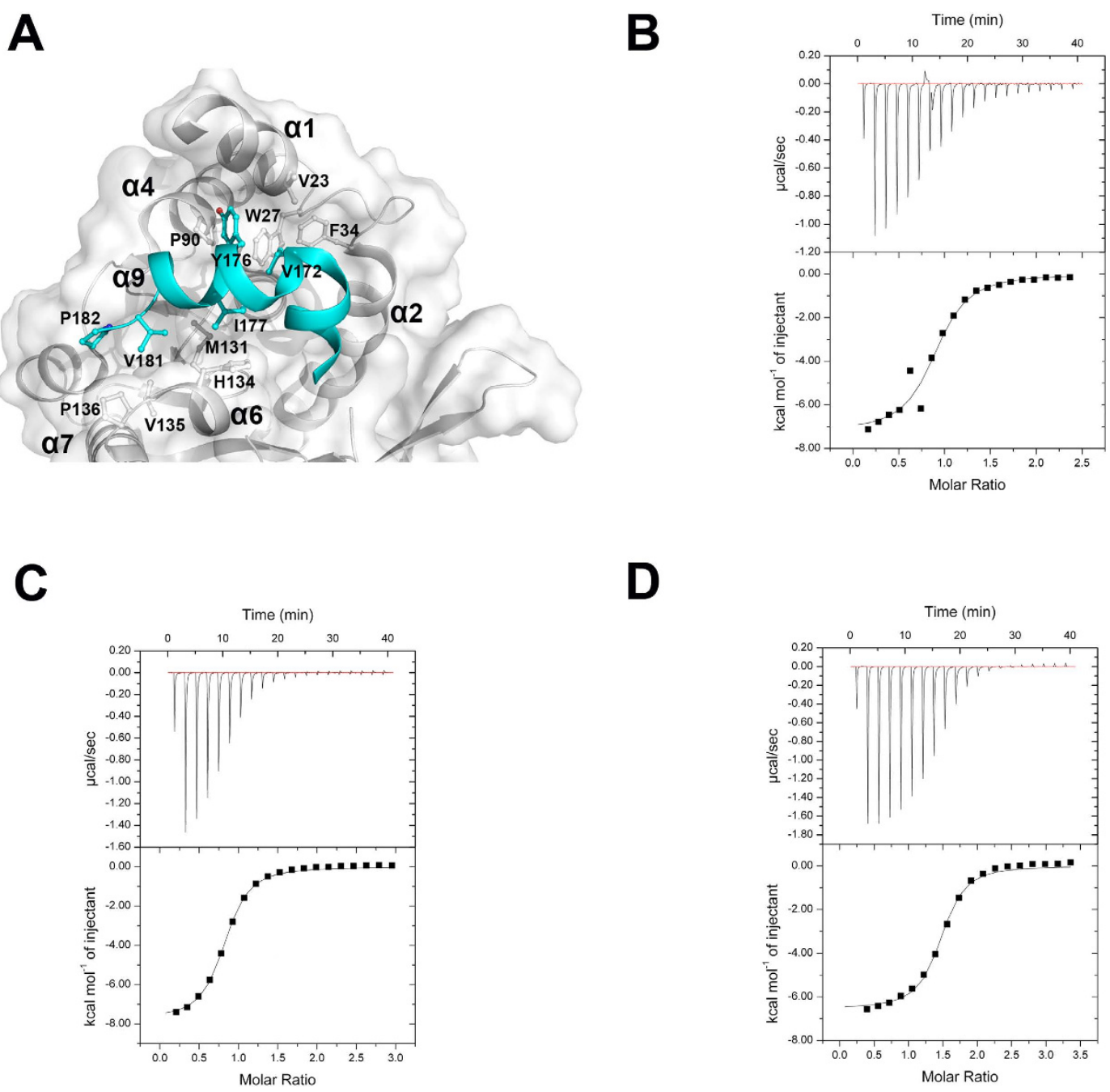

Figure 2. The membrane binding region of UbiG gates the entrance of methyl donor. (A) Surface show of the structure of UbiG. The insertions of structural elements in UbiG are colored cyan. Residues involved in the hydrophobic interaction network of $\alpha 9$ with the core structure of UbiG are labelled. ITC profile of SAH titrated against UbiG-M1 (B), UbiG-M2 (C) and UbiG $\Delta^{165-187}($ D).

the methyl donor diffusion mechanism of UbiG, and reveal that the membrane association of UbiG may regulate the entrance of methyl donor, which suggests an inextricable relationship between membrane anchoring and $O$-methyl transfer reaction in the ubiquinone biosynthesis pathway.

\section{Results and Discussion}

UbiG bound with liposome displays an enhanced binding ability toward SAH. Our previous results have reported the crystal structure of UbiG from $E$. coli, and identified the residues vital for membrane binding. Interestingly, these residues mainly locate in helix $\alpha 9$ and loop $\alpha 9 / \alpha 10$, a region that covers the possible methyl donor binding pocket ${ }^{15}$. Moreover, to gain insight into the methyl donor recognition model of UbiG, we tried to determine the complex structure of UbiG with SAH. However, we failed to obtain the complex structure by either co-crystallization or crystal soaking. To investigate whether the membrane association of UbiG influences the diffusion of methyl donor, we compare the binding affinity of wild-type UbiG and liposome-bound UbiG to SAH (Table 2). The ITC experiments show that wild-type UbiG bound SAH with a $K_{\mathrm{d}}$ of $104.43 \pm 17.21 \mu \mathrm{M}$ (Fig. 1B), whereas the affinity of liposome-bound UbiG to SAH $\left(K_{d}=9.63 \pm 2.10 \mu \mathrm{M}\right)$ increased $\approx 11$-fold (Fig. $1 \mathrm{C}$ ), indicating that the membrane association promotes UbiG interacting with SAH.

The membrane binding region of UbiG gates the entrance of methyl donor. In the structure of UbiG, the membrane binding region including $\alpha 9$ and loop $\alpha 9 / \alpha 10$ is stabilized by hydrophobic interactions with the core structure. As shown in Fig. 2(A), residues Val ${ }^{172}$ (helix $\alpha 9$ ), $\operatorname{Tyr}^{176}$ (helix $\alpha 9$ ), Ile ${ }^{177}$ (helix $\alpha 9$ ), Val ${ }^{181}$ (loop $\alpha 9 / \alpha 10$ ) and Pro $^{182}$ (loop $\alpha 9 / \alpha 10$ ) make extensive hydrophobic contacts with residues Val ${ }^{23}$ (helix $\alpha 1$ ), $\operatorname{Trp}^{27}$ (loop $\alpha 1 / \alpha 2$ ), Phe $^{34}$ (helix $\alpha 2$ ), Pro ${ }^{90}$ (helix $\alpha 4$ ), Met $^{131}$ (helix $\alpha 6$ ), His ${ }^{134}$ (helix $\alpha 6$ ), Val ${ }^{135}$ (helix $\alpha 6$ ), and Pro $^{136}$ (loop $\alpha 6 / \alpha 7$ ) of the core structure of UbiG. To investigate whether this membrane binding region affects 


\begin{tabular}{|l|c|}
\hline Data collection statistics & UbiG $\Delta^{165-187}-\mathrm{SAH}$ \\
\hline Space Group & $\mathrm{C} 2$ \\
\hline Unit Cell Parameters & $139.8,39.3,40.1$ \\
\hline$a, b, c(\AA)$ & $90.0,94.3,90.0$ \\
\hline$\alpha, \beta, \gamma\left({ }^{\circ}\right)$ & 0.9792 \\
\hline Wavelength $(\AA)$ & $50.00-2.10(2.18-2.10)$ \\
\hline${ }^{\mathrm{A}}$ Resolution limits $(\AA)$ & 12887 \\
\hline No. of unique reflections & $99.2(99.2)$ \\
\hline Completeness $(\%)$ & $3.5(3.3)$ \\
\hline Redundancy & $14.4(63.3)$ \\
\hline${ }^{\mathrm{B}} R_{\text {merge }}(\%)$ & $9.0(41.1)$ \\
\hline$R_{\text {p.i.m }}(\%)$ & $11.9(3.0)$ \\
\hline Mean $\mathrm{I} / \sigma(\mathrm{I})$ & \\
\hline Refinement Statistics & $50.00-2.10$ \\
\hline Resolution limits $(\AA)$ & $17.63 / 21.52$ \\
\hline${ }^{\mathrm{C}} R_{\text {work }}(\%) /{ }^{\circ} R_{\text {free }}(\%)$ & 0.008 \\
\hline Rmsd for bonds $(\AA)$ & 1.095 \\
\hline Rmsd for angles $\left({ }^{\circ}\right)$ & 22.73 \\
\hline B factor $\left(\AA^{2}\right)$ & 30.04 \\
\hline Protein & \\
\hline Water & 20.70 \\
\hline SAH & 1552 \\
\hline No. of non-hydrogen protein atoms & 86 \\
\hline No. of water oxygen atoms & 91.9 \\
\hline Ramachandran plot $(\%)$ & $5 \mathrm{DPM}$ \\
\hline most favored regions & \\
\hline additional allowed regions & \\
\hline generously allowed regions & \\
\hline PDB entry & \\
\hline & \\
\hline
\end{tabular}

Table 1. Data collection and Refinement Statistics for UbiG $\Delta^{165-187}$ in complex with SAH. ${ }^{A}$ Values in parentheses are for the highest resolution shell. ${ }^{\mathrm{B}} R_{\text {merge }}=\Sigma \mathrm{h} \Sigma \mathrm{l}|\mathrm{Ihl}-<\mathrm{Ih}\rangle|/ \Sigma \mathrm{h} \Sigma \mathrm{l}<\mathrm{Ih}\rangle$, where Ihl is the lth observation of reflection $\mathrm{h}$ and $<\mathrm{Ih}>$ is the weighted average intensity for all observations $\mathrm{l}$ of reflection $\mathrm{h}$. ${ }^{\mathrm{C}} R_{\text {work }}$ factor $=\Sigma \mathrm{h}||$ Fobs $(\mathrm{h})|-| \mathrm{Fcal}(\mathrm{h})|| / \Sigma \mathrm{h} \mid$ Fobs(h)|, where Fobs(h) and Fcal(h) are the observed and calculated structure factors for reflection $\mathrm{h}$ respectively. ${ }^{\mathrm{D}} R_{\text {free }}$ factor was calculated same as $R_{\text {work }}$ factor using the $5 \%$ the reflections selected randomly and omitted from refinement.

the diffusion of methyl donor, we construct UbiG mutants to enhance the flexibility of this region. We designed two UbiG mutants, UbiG-M1 (residues Val ${ }^{172}$ and Tyr ${ }^{176}$ mutated to Ala) and UbiG-M2 (residues Ile ${ }^{177}$, Val ${ }^{181}$ and Pro ${ }^{182}$ mutated to Ala) to weaken the interaction of this membrane binding region with the core structure of UbiG. The ITC experiments show that the binding affinity of UbiG-M1 to SAH was $3.37 \pm 0.84 \mu \mathrm{M}$, increasing $\approx 31$-fold compared with that of wild-type UbiG (Fig. $2 \mathrm{~B}$ ). UbiG-M2 bound SAH with a $K_{\mathrm{d}}$ of $2.77 \pm 0.36 \mu \mathrm{M}$, increasing $\approx 38$-fold compared with that of wild-type UbiG (Fig. 2C). To further confirm our hypothesis, we designed another UbiG mutant (UbiG $\Delta^{165-187}$ ) by deleting this membrane interacting region that covers the methyl donor binding pocket. We compared the binding affinity of wild-type UbiG and UbiG $\Delta^{165-187}$ to SAH by ITC experiments. The binding affinity of UbiG $\Delta^{165-187}$ to SAH was $1.84 \pm 0.16 \mu \mathrm{M}$, increasing $\approx 57$-fold compared with that of wild-type UbiG (Fig. 2D). These data strongly support our hypothesis, and confirm that in the membrane-unbound state, the membrane binding region of UbiG hinds the entrance of methyl donor.

Structure of UbiG $\Delta^{\mathbf{1 6 5 - 1 8 7}}$ in complex with SAH. To disclose the accurate recognition pattern of SAH, we crystallized UbiG $\Delta^{165-187}$ in complex with SAH at a resolution of $2.10 \AA$. The details of the data collection and refinement statistics are summarized in Table 1 . The final model contains one molecule of UbiG $\Delta^{165-187}$ and one molecule of SAH, with a stoichiometry of 1:1. Due to the insufficient electron density, the N-terminal 9 residues could not be traced. UbiG $\Delta^{165-187}$ displays a similar fold as wild-type UbiG (Fig. 3A). The overall main-chain root-mean squared deviation (RMSD) between UbiG $\Delta^{165-187}$ and wild-type UbiG is $0.397 \AA$ for 215 comparable $\mathrm{C} \alpha$ atoms. Comparison with the structure of wild-type UbiG, helix $\alpha 1$ of UbiG $\Delta^{165-187}$ moves toward the SAH binding pocket and forms extensive hydrophobic interactions with the carbon-skeleton of SAH (Fig. 3B). In addition, due to the lack of the hydrophobic packing with helix $\alpha 8$, the $\beta 6$ and $\beta 7$ of UbiG $\Delta^{165-187}$ move away from the core structure (Fig. 3B).

The electron density for the SAH is well defined in the final model of UbiG $\Delta^{165-187}$ and the SAH is bound via an extensive hydrogen bond network and hydrophobic interaction. In light of the structure, we easily identify the $\mathrm{SAH}$ binding sites. The adenine ring of SAH is located in a hydrophobic pocket constituted by residues $\mathrm{Val}^{12}, \mathrm{Ile}^{17}$, 


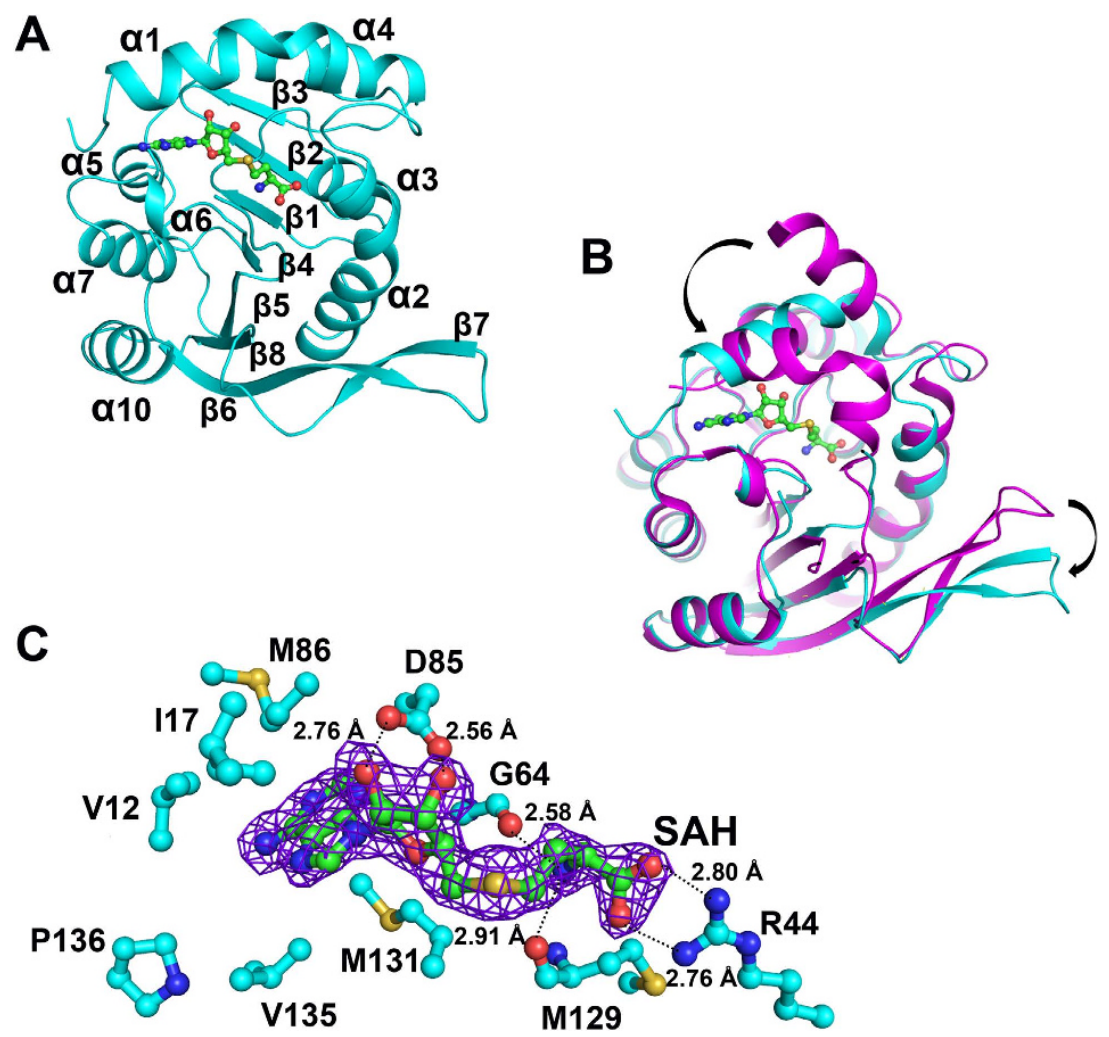

Figure 3. Structure of UbiG $\Delta^{\mathbf{1 6 5 - 1 8 7}}$ in complex with SAH. (A) Cartoon show of the overall structure of UbiG $\Delta^{165-187}$ in complex with SAH. The $\alpha$-helices and $\beta$ strands are labelled and colored cyan. The methyl donor product SAH is shown as a ball-and-stick model and is colored green. (B) Superimposition of the structures of wild-type UbiG and UbiG $\Delta^{165-187}$ in complex with SAH. Wild-type UbiG and UbiG $\Delta^{165-187}$ are colored magenta and cyan, respectively. (C) SAH binding model of UbiG $\Delta^{165-187}$. The 2Fo-Fc electron density map (contoured at $1 \sigma$ ) for SAH is shown as blue. The residues involved in interacting with SAH of UbiG $\Delta^{165-187}$ are labelled and colored cyan. The dashed lines represent hydrogen bonds.

$\mathrm{Met}^{86}, \mathrm{Met}^{131}$, Val ${ }^{135}$, and Pro $^{136}$ (Fig. 3C). The ribosyl moiety is anchored via hydrogen bonds from the O2' and $\mathrm{O}^{\prime}$ hydroxyl groups to the side chain of Asp ${ }^{85}$ (Fig. 3C). The SAH carboxyl is locked by the side chain of $\mathrm{Arg}^{44}$, whereas the corresponding SAH amine is anchored to the main chain carbonyl oxygen atoms of Gly ${ }^{64}$ and Met ${ }^{129}$ via hydrogen bonds (Fig. 3C).

The methyl donor binding model and diffusion mechanism of UbiG. Superimposition of the structures of wild-type UbiG and UbiG $\Delta^{165-187}$ in complex with SAH, we map the SAH binding model of wild-type UbiG. As shown in Fig. 4(A), SAH is situated in the central cavity of the Rossmann-fold domain of UbiG. The interaction between UbiG and SAH can be divided into three parts in accordance to the moieties of SAH. For the adenine moiety, hydrophobic residues $\mathrm{Met}^{86}$, $\mathrm{Met}^{131}$, Val ${ }^{135}$, $\mathrm{Met}^{180}$, $\mathrm{Val}^{181}$ and Pro ${ }^{182}$ make extensive van der Waals interactions with the adenine ring (Fig. 4A). For the ribosyl moiety, the side chain of Asp ${ }^{85}$ forms two hydrogen bonds with the $\mathrm{O}^{\prime}$ and $\mathrm{O}^{\prime}$ ' hydroxyl groups (Fig. 4A). The interaction between UbiG and the homocysteine moiety of SAH is dominated by four hydrogen bonds. The side-chain of $\mathrm{Arg}^{44}$ contributes to two hydrogen bonds with the amino group of the homocysteine (Fig. 4A). The carboxyl group of the homocysteine makes another two hydrogen bonds with the main-chain carbonyl oxygen atoms of Gly ${ }^{64}$ and $\operatorname{Met}^{129}$, respectively (Fig. 4A). Then, we used the program CAVER to analyse the diffusion pathway of the methyl donor, which revealed a tunnel gated by residues $\mathrm{Met}^{86}$, $\mathrm{Thr}^{111}, \mathrm{Glu}^{113}$, $\mathrm{Pro}^{136}, \mathrm{Asp}^{137}, \mathrm{Ser}^{140}$, and $\mathrm{Pro}^{182}$ (Fig. 4B). This gate seems much narrow compared with that of most other class I SAM-MTases, such as catechol $O$-methyltransferase COMT (PDB code $1 \mathrm{VID})^{16}$, rebeccamycin sugar $4^{\prime}$-O-Methyltransferase RebM (PDB code 3BUS) ${ }^{17}$, and 2-methoxy-6-polyprenyl-1, 4-benzoquinone $5^{\prime}$-C-methyltransferase Coq5 $(\mathrm{PDB} \text { code } 4 \mathrm{OBX})^{18}$, in which the methyl donor binding pocket is uncovered.

Combining with the ITC results mentioned above, we conclude that in the membrane-unbound state, the diffusion of methyl donor of UbiG is greatly affected by the narrow gate constituted by the membrane binding region. When UbiG associates with the membrane, strong hydrophobic driving forces may loosen the interaction of this membrane binding region with the core structure, and cause a relatively open channel for the diffusion of methyl donor during the $O$-methyl transfer process for ubiquinone biosynthesis (Fig. 4C). Association of membrane-bound proteins with the surface of cellular membranes usually plays a necessary role for a large variety of cellular functions. For example, the cytoskeleton uses the lipid-binding domain for directly anchoring to the membrane surface ${ }^{19}$. Bin-Amphiphysin-Rvs (BAR) domain containing proteins bind to the membrane 

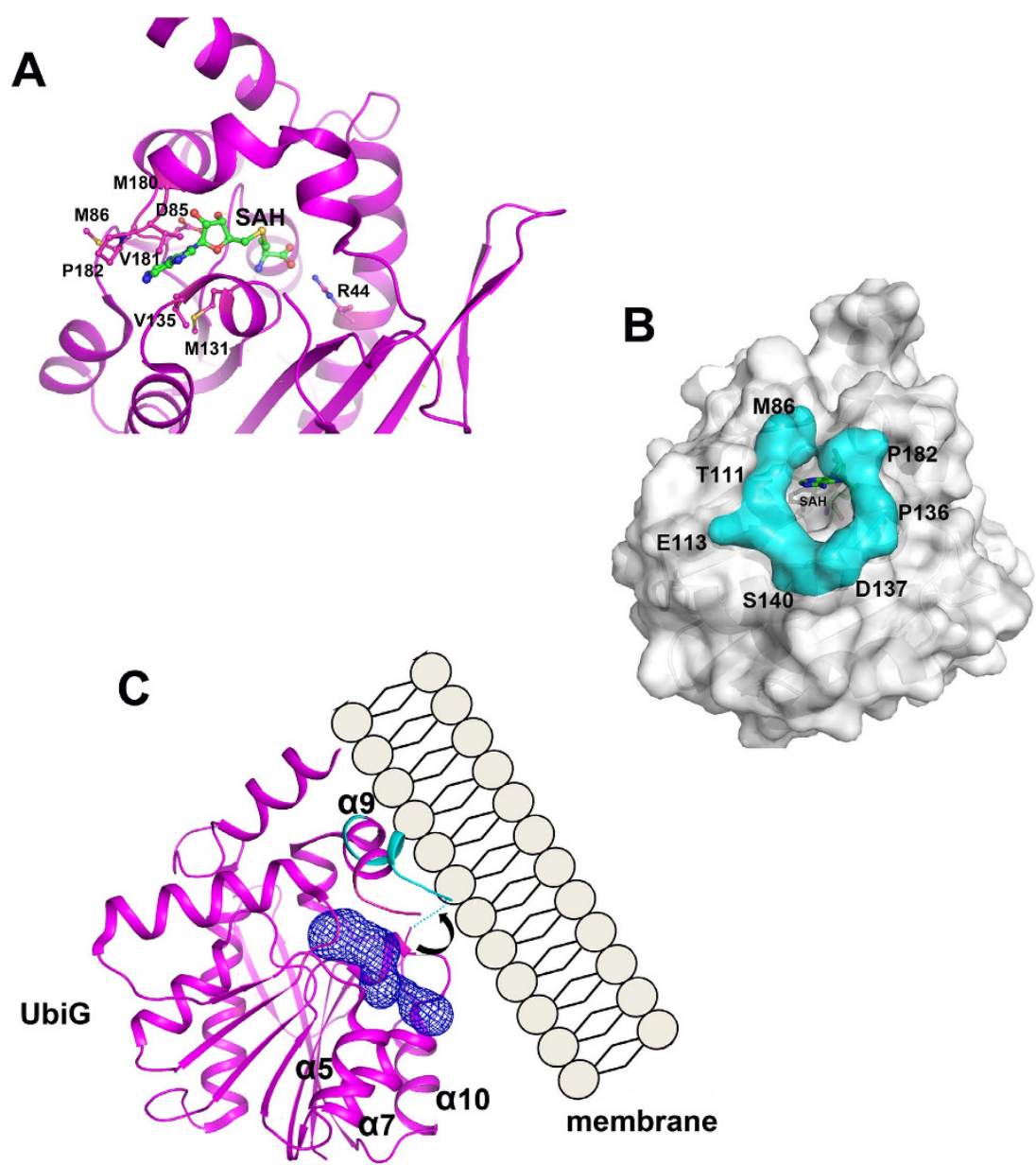

Figure 4. The methyl donor binding model and diffusion mechanism of UbiG. (A) A proposed SAH recognition model of UbiG. Residues involved in the interaction with SAH are labelled. (B) Surface show of the structure of UbiG. The residues gated the diffusion of the methyl donor are labelled and colored cyan. (C) Cartoon representation of the methyl donor diffusion mechanism of UbiG. Putative SAH access tunnel is calculated by CAVER and is denoted in mesh (blue). When UbiG associates with the membrane, strong hydrophobic driving forces may loosen the interaction of this membrane binding region with the core structure, and cause a relatively open channel for the diffusion of methyl donor during the O-methyl transfer process for ubiquinone biosynthesis.

surface to act as membrane shapers ${ }^{20}$. The attaching of alpha-toxin to membrane surface pushes the opening of the active center, which is help for hydrolysis of membrane phospholipids ${ }^{21,22}$. As we known, the $O$-methyl transfer reaction for ubiquinone biosynthesis catalyzed by $\mathrm{UbiG}$ is membrane associate in vivo ${ }^{14}$. Obviously, the membrane anchoring ability of UbiG is of benefit for sequestering substrates located in the lipid bilayer. In this study, we find surprisingly that the membrane association of UbiG also regulates the entrance of methyl donor, thus activating the $O$-methyl transfer reaction for ubiquinone biosynthesis. Our results provide much insight into the role of membrane association in regulating the enzyme activity of UbiG, and enhance our better understanding of the $\mathrm{O}$-methyl transfer process for ubiquinone biosynthesis in vivo.

\section{Materials and Methods}

Cloning, expression and purification. Full-length UbiG from E. coli was expressed and purified as described previously ${ }^{23}$. UbiG mutants was generated by PCR with the MutanBEST Kit (TaKaRa) using the parent expression plasmid pET28a-UbiG (1-240) as template. The mutant plasmids were confirmed by DNA sequencing (Invitrogen). Plasmids containing the confirmed UbiG mutations were then transformed into E. coli BL21 (DE3) strain (Novagen), and the corresponding overproduced recombinant mutant proteins were purified as described for the wild-type $\mathrm{UbiG}^{23}$.

Crystallization, data collection and processing. Crystallization trials were conducted using the hanging drop vapour diffusion method at $287 \mathrm{~K}$. The protein UbiG $\Delta^{165-187}$ was concentrated to approximately $16 \mathrm{mg} / \mathrm{ml}$. The UbiG $\Delta^{165-187}$-SAH complex was prepared by mixing UbiG $\Delta^{165-187}$ with SAH at a 1:3 molar ratio. Diffraction quality crystals of UbiG $\Delta^{165-187}$-SAH complex were obtained with $0.1 \mathrm{M}$ citric acid pH 5.0 and $20 \%$ (v/v) 2-Methyl-2,4-pentanediol. For data collection, the crystals were cryo-protected using 25\% (v/v) glycerol 


\begin{tabular}{|c|c|c|c|c|c|}
\hline \multirow[b]{2}{*}{ Proteins } & $K_{\mathrm{D} 1}$ & $K_{\mathrm{D} 2}$ & $K_{\mathrm{D} 3}$ & $\bar{K}_{\mathrm{D}}$ & \multirow{2}{*}{$\begin{array}{l}\text { Standard } \\
\text { Deviation }\end{array}$} \\
\hline & $\mu \mathrm{M}$ & $\mu \mathrm{M}$ & $\mu \mathrm{M}$ & $\mu \mathrm{M}$ & \\
\hline wt-UbiG & $101.18 \pm 11.17$ & $103.91 \pm 18.80$ & $108.20 \pm 21.67$ & $104.43 \pm 17.21$ & 2.89 \\
\hline wt-UbiG with liposome & $9.14 \pm 1.63$ & $9.09 \pm 2.06$ & $10.65 \pm 2.59$ & $9.63 \pm 2.10$ & 0.72 \\
\hline UbiG-M1 & $3.29 \pm 1.10$ & $3.48 \pm 0.69$ & $3.34 \pm 0.73$ & $3.37 \pm 0.84$ & 0.08 \\
\hline UbiG-M2 & $2.47 \pm 0.19$ & $3.27 \pm 0.59$ & $2.56 \pm 0.30$ & $2.77 \pm 0.36$ & 0.36 \\
\hline UbiG $\Delta^{165-187}$ & $1.73 \pm 0.24$ & $1.73 \pm 0.09$ & $2.05 \pm 0.13$ & $1.84 \pm 0.16$ & 0.15 \\
\hline
\end{tabular}

Table 2. The thermodynamic parameters of the ITC experiments.

supplemented with crystallization solution, and flashed cool in liquid nitrogen. Diffraction data sets for the UbiG $\Delta^{165-187}$-SAH complex were collected on beamline 19U of the Shanghai Synchrotron Radiation Facility (SSRF) using a CCD detector. All frames were collected at $100 \mathrm{~K}$ using a $1^{\circ}$ oscillation angle with an exposure time of $0.2 \mathrm{~s}$ per frame. The crystal-to-detector distance was set to $250 \mathrm{~mm}$. The complete diffraction datasets were subsequently processed using $H K L-2000^{24}$ and programs in CCP4 package ${ }^{25}$. To capture an open state of UbiG, we prepared UbiG-phosphatidylglycerol (PG) complex by mixing $16 \mathrm{mg} / \mathrm{ml}$ protein with PG in a molecular ratio of 1:3 1:10. Crystallization screens were performed with a Mosquito liquid-handling robot (TTP LabTech) using the vapour-diffusion method in 96 -well crystallization plates at $289 \mathrm{~K}$. We also tried to screen UbiG for other crystal morphologies as an alternative. However, both of these attempts were failed.

Structure determination and refinement. The complex structure of the UbiG $\Delta^{165-187}$-SAH was solved using the molecular replacement method in Molrep ${ }^{26}$, using the structure of the full-length UbiG from $E$. coli K12 (PDB code 4KDC) as the search model. The model was refined at $2.10 \AA$ A resolution using Refmac5 ${ }^{27}$ and $C O O T^{28}$ by manual model correction. The structure factors refinement were converged to an R-factor of $17.63 \%$ and R-free of $21.52 \%$. These final models were both evaluated with the programs MOLPROBITYY ${ }^{29}$ and PROCHECK ${ }^{30}$. The data collection and structure refinement statistics were listed in Table 1. All structure figures were created using the program PyMol (DeLano Scientific LLC).

Liposome preparation. The total lipid extract of E. coli (Avanti Polar Lipids, Inc) was used to generate liposomes that mimic the component of the E. coli plasma membrane. For liposome preparation, the total lipid extract were dissolved in chloroform in a glass tube and then was evaporated under a stream of nitrogen for 20 minutes. Next, the lipid films were dried with a vacuum pump overnight and then were hydrated at room temperature with constant mixing in buffer $(20 \mathrm{mM}$ Tris- $\mathrm{HCl}, 50 \mathrm{mM} \mathrm{NaCl}, \mathrm{pH} 7.5)$. After hydration, lipid vesicles were subjected to freeze-thaw cycles in liquid nitrogen and a room temperature water bath, and then sized using Mini-Extruder Set (Avanti) with $100 \mathrm{~nm}$ polycarbonate filters.

Isothermal titration calorimetry (ITC) experiments. The ITC binding studies were performed using an ITC200 (GE) at room temperature with $0.04 \mathrm{ml}$ of $1 \mathrm{mM} \mathrm{SAH}$ in the injector cell and $0.26 \mathrm{ml}$ of $2 \mathrm{mg} / \mathrm{mL}$ $(75 \mathrm{mM}) \mathrm{UbiG}, \mathrm{UbiG}$ mutants and liposome-bound UbiG in the sample cell, respectively. The protein and ligands were kept in a buffer consisting of $20 \mathrm{mM}$ Tris- $\mathrm{HCl}(\mathrm{PH} \mathrm{7.5)}$ and $50 \mathrm{mM} \mathrm{NaCl}$. Five group experiments were conducted: for the first four groups, proteins (wt-UbiG and three UbiG mutants, respectively) were titrated with SAH directly, and for another group, wt-UbiG was titrated after the incubation with liposome. For the preparation of UbiG and liposome complex, $400 \mu \mathrm{g}$ liposome was incubated with UbiG at $4{ }^{\circ} \mathrm{C}$ for $30 \mathrm{~min}$. Twenty microliters injection volumes were used for all experiments. Two consecutive injections were separated by 2 min to reset the baseline. The control experiment, consisting of titration of SAH against buffer, was performed and substracted from each experiment to adjust for the heat of dilution of ligands. ITC data was analyzed with a single-site fitting model, using Origin 8.6 (OriginLab Corp).

Analysis of methyl donor entrance. The software of CAVER was used to explore the putative cofactor access tunnel of UbiG. The position of SAH in the interior pocket was specified to identify tunnels directly connecting the cofactor binding site to the surface. The tunnel profile, which was the average tunnel cross-section radius along the length, was calculated from the detected accessible path.

\section{References}

1. Brandt, U. \& Trumpower, B. The protonmotive Q cycle in mitochondria and bacteria. Crit Rev Biochem Mol Biol 29, 165-97 (1994).

2. Turunen, M., Olsson, J. \& Dallner, G. Metabolism and function of coenzyme Q. Biochimica et Biophysica Acta (BBA)-Biomembranes 1660, 171-199 (2004).

3. Mitchell, P. Possible molecular mechanisms of the protonmotive function of cytochrome systems. J Theor Biol 62, 327-67 (1976).

4. Beal, M. F. Mitochondrial dysfunction and oxidative damage in Alzheimer's and Parkinson's diseases and coenzyme Q10 as a potential treatment. J Bioenerg Biomembr 36, 381-6 (2004).

5. Laredj, L. N., Licitra, F. \& Puccio, H. M. The molecular genetics of coenzyme Q biosynthesis in health and disease. Biochimie 100, 78-87 (2014).

6. Garrido-Maraver, J. et al. Clinical applications of coenzyme Q10. Front Biosci (Landmark Ed) 19, 619-33 (2014).

7. Kawamukai, M. Biosynthesis and bioproduction of coenzyme Q10 by yeasts and other organisms. Biotechnol Appl Biochem 53, 217-26 (2009).

8. Bentinger, M., Tekle, M. \& Dallner, G. Coenzyme Q-biosynthesis and functions. Biochem Biophys Res Commun 396, 74-9 (2010).

9. Aussel, L. et al. Biosynthesis and physiology of coenzyme Q in bacteria. Biochim Biophys Acta 1837, 1004-1011 (2014). 
10. Hsu, A. Y., Poon, W. W., Shepherd, J. A., Myles, D. C. \& Clarke, C. F. Complementation of coq3 mutant yeast by mitochondrial targeting of the Escherichia coli UbiG polypeptide: evidence that UbiG catalyzes both O-methylation steps in ubiquinone biosynthesis. Biochemistry 35, 9797-806 (1996).

11. Fontecave, M., Atta, M. \& Mulliez, E. S-adenosylmethionine: nothing goes to waste. Trends Biochem Sci 29, 243-9 (2004).

12. Schubert, H. L., Blumenthal, R. M. \& Cheng, X. Many paths to methyltransfer: a chronicle of convergence. Trends in Biochemical Sciences 28, 329-335 (2003).

13. Poon, W. W. et al. Yeast and rat Coq3 and Escherichia coli UbiG polypeptides catalyze both O-methyltransferase steps in coenzyme Q biosynthesis. J Biol Chem 274, 21665-72 (1999).

14. Leppik, R. A., Stroobant, P., Shineberg, B., Young, I. G. \& Gibson, F. Membrane-associated reactions in ubiquinone biosynthesis. 2-Octaprenyl-3-methyl-5-hydroxy-6-methoxy-1,4-benzoquinone methyltransferase. Biochim Biophys Acta 428, 146-56 (1976).

15. Zhu, Y. et al. Structural and biochemical studies reveal UbiG/Coq3 as a class of novel membrane-binding proteins. Biochem J 470, 105-14 (2015).

16. Vidgren, J., Svensson, L. A. \& Liljas, A. Crystal-Structure of Catechol O-Methyltransferase. Nature 368, 354-358 (1994).

17. Singh, S. et al. Structure and mechanism of the rebeccamycin sugar 4'-O-Methyltransferase RebM. Journal of Biological Chemistry 283, 22628-22636 (2008)

18. Dai, Y. N. et al. Crystal structures and catalytic mechanism of the C-methyltransferase Coq5 provide insights into a key step of the yeast coenzyme Q synthesis pathway. Acta Crystallogr D Biol Crystallogr 70, 2085-92 (2014).

19. Skruzny, M. et al. Molecular basis for coupling the plasma membrane to the actin cytoskeleton during clathrin-mediated endocytosis. Proc Natl Acad Sci USA 109, E2533-42 (2012).

20. Kessels, M. M. \& Qualmann, B. Different functional modes of BAR domain proteins in formation and plasticity of mammalian postsynapses. J Cell Sci 128, 3177-85 (2015).

21. Titball, R. W., Naylor, C. E., Miller, J., Moss, D. S. \& Basak, A. K. Opening of the active site of Clostridium perfringens alpha-toxin may be triggered by membrane binding. Int J Med Microbiol 290, 357-61 (2000).

22. Eaton, J. T. et al. Crystal structure of the C. perfringens alpha-toxin with the active site closed by a flexible loop region. J Mol Biol 319, 275-81 (2002).

23. Xing, L. et al. Crystallization and preliminary crystallographic studies of UbiG, an O-methyltransferase from Escherichia coli. Acta Crystallogr Sect F Struct Biol Cryst Commun 67, 727-9 (2011).

24. Otwinowski, Z. \& Minor, W. Processing of X-ray diffraction data collected in oscillation mode. Macromolecular Crystallography, Pt A 276, 307-326 (1997).

25. The CCP4 suite: programs for protein crystallography. Acta Crystallogr D Biol Crystallogr 50, 760-3 (1994).

26. Vagin, A. \& Teplyakov, A. An approach to multi-copy search in molecular replacement. Acta Crystallogr D Biol Crystallogr 56, $1622-4(2000)$.

27. Murshudov, G. N. et al. REFMAC5 for the refinement of macromolecular crystal structures. Acta Crystallogr D Biol Crystallogr 67, 355-67 (2011).

28. Emsley, P. \& Cowtan, K. Coot: model-building tools for molecular graphics. Acta Crystallogr D Biol Crystallogr 60, 2126-32 (2004).

29. Davis, I. W. et al. MolProbity: all-atom contacts and structure validation for proteins and nucleic acids. Nucleic Acids Res 35, W375-83 (2007)

30. Laskowski, R. A., Rullmannn, J. A., MacArthur, M. W., Kaptein, R. \& Thornton, J. M. AQUA and PROCHECK-NMR: programs for checking the quality of protein structures solved by NMR. J Biomol NMR 8, 477-86 (1996).

\section{Acknowledgements}

We thank the staff of BL17B/BL18U1/BL19U1 beamlines at National Center for Protein Sciences Shanghai and Shanghai Synchrotron Radiation Facility, Shanghai, People's Republic of China, for assistance during data collection. Financial support for this project was provided by the Chinese National Natural Science Foundation (Grant No. 31130018), the Chinese Ministry of Science and Technology (Grant No. 2012CB917200), the Chinese National Natural Science Foundation (Grant Nos 31370732, 31270014 and U1432107), the Scientific Research Grant of Hefei Science Center of CAS (Grant No. 2015SRG-HSC042).

\section{Author Contributions}

Y.Z. and X.J. designed and conducted the experiments, and wrote the paper. C.W., Y.L., X.F. and L.Z. conducted the experiments. L.N. contributed to the experimental design. X.L. and M.T. co-ordinated the experimental work, and contributed to the experimental design and writing of the paper.

\section{Additional Information}

Data availability : The coordinates and structure factors of UbiG $\Delta$ 165-187-SAH complex were deposited in the Protein Data Bank with the access code 5DPM.

Competing financial interests: The authors declare no competing financial interests.

How to cite this article: Zhu, Y. et al. Structural insights into the methyl donor recognition model of a novel membrane-binding protein UbiG. Sci. Rep. 6, 23147; doi: 10.1038/srep23147 (2016).

This work is licensed under a Creative Commons Attribution 4.0 International License. The images or other third party material in this article are included in the article's Creative Commons license, unless indicated otherwise in the credit line; if the material is not included under the Creative Commons license, users will need to obtain permission from the license holder to reproduce the material. To view a copy of this license, visit http://creativecommons.org/licenses/by/4.0/ 